\title{
Operation of the ATLAS Semiconductor Tracker: commissioning and performance results with cosmic ray data
}

\author{
Gonzalez-Sevilla, S.,a, on behalf of the ATLAS Collaboration \\ ${ }^{a}$ DPNC, Université de Genève, 24 Quai Ernest-Ansermet, 1211 Genève 4 (Suisse)
}

\begin{abstract}
The Semiconductor Tracker (SCT) is one of the three sub-systems of the ATLAS internal tracker. Its complete installation and sign-off took about 18 months and was finished at the beginning of 2008. Since then, the SCT has been run successfully taking data in combined mode with the other ATLAS sub-systems. The major problems related with cooling failures and the mortality of off-detector opto-chips have been solved. As in summer 2009, more than $99 \%$ of the main detector components are fully working. Detailed calibration procedures have been applied to optimize the detector performance. An initial alignment has been achieved using cosmic ray real data. Some results in terms of tracking performance and Lorentz angle measurements are also shown.
\end{abstract}

Key words: ATLAS; SCT; Semiconductor; Silicon; Tracker; Commissioning

\section{Introduction}

The Large Hadron Collider (LHC) is a proton-proton collider installed at CERN, near Geneva (Switzerland). The beams will collide at a center-of-mass energy of $\sqrt{s}=14 \mathrm{TeV}$ with a bunch-crossing rate of $40 \mathrm{MHz}$. The ATLAS detector [1] is a general-purpose experiment designed to fully exploit the physics potential of the LHC at the designed luminosity of $10^{34} \mathrm{~cm}^{-2} \mathrm{~s}^{-1}$.

The Inner Detector (ID) is the ATLAS internal tracker. It combines high-resolution discrete silicon detectors (pixel and microstrip detectors) in the innermost layers with a continous gaseous straw drift-tube detector in the outermost radii. This layout ensures a robust pattern recognition, momentum and charge determination, precise vertex measurements and some particle identification capabilities (electron/pion separation). The ID (see Fig. 1) is composed of three sub-systems (from the inside-out): the Pixel detector, the Semicondutor Tracker (SCT) and the Transition Radiation Tracker (TRT). It provides efficient track reconstruction over the pseudo-rapidity range $|\eta| \leq 2.5$. A central superconducting solenoid surrounding the TRT provides a $2 \mathrm{~T}$ axial field for bending the charged particles.

\footnotetext{
${ }^{*}$ Corresponding author

Email address: Sergio.Gonzalez.Sevilla@cern.ch (Gonzalez-Sevilla, S.)

Preprint submitted to Elsevier
}

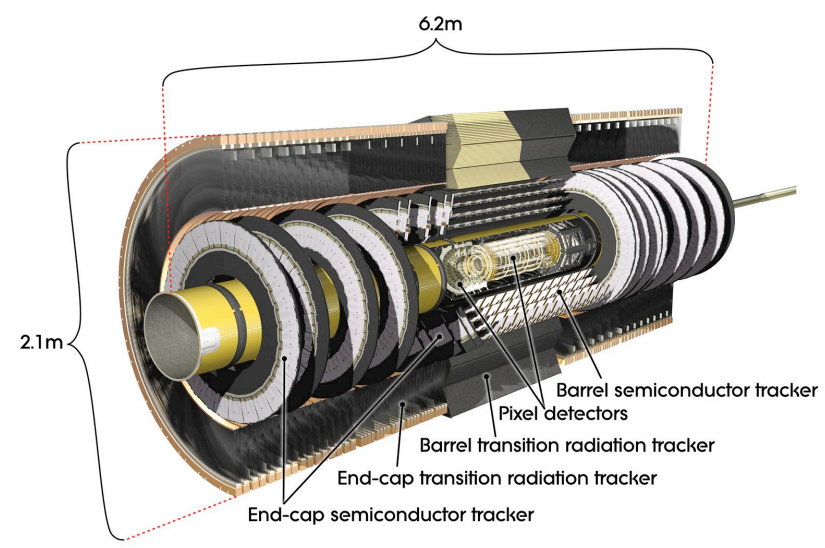

Figure 1: View of the ATLAS Inner Detector.

\section{The Semiconductor Tracker}

The Semiconductor Tracker (SCT) has been designed to provide four precision space-point measurements per track at intermediate radii of the ID. It consists of a barrel region and two symmetric end-caps. The barrel SCT is made of 2112 identical barrel modules arranged in four nested cylindrical layers located at average radii of 299, 371, 443 and $514 \mathrm{~mm}$. Each of the two end-caps has nine disks, with 988 forward modules arranged in rings within each disk. The sensitive volume extends September 24, 2009 
in $|Z|$ (direction along the beam-axis) from 839 to 2735 $\mathrm{mm}$, and in radius from 275 to $560 \mathrm{~mm}$.

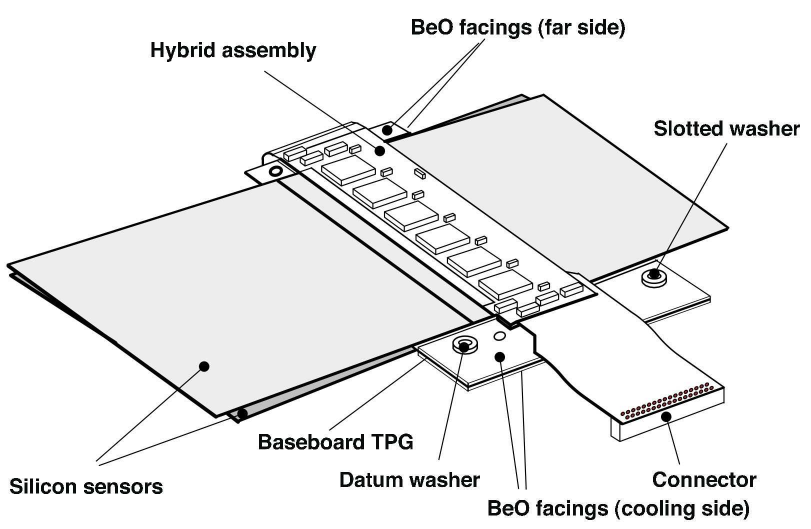

Figure 2: Schematic view of the SCT barrel module.

The basic detecting unit in the SCT is the module. Both barrel and end-cap modules have identical components but just differ in shape. In most cases a module (see Fig. 2) consists of four silicon microstrips detectors glued by pairs on top and bottom of a base-board at an angle of $40 \mathrm{mrad}$ to make a stereo measurement. The silicon sensor is p-on-n with 768 channels and a strippitch of $80 \mu \mathrm{m}$ in the barrel and varying from $\sim 55$ to $\sim 90 \mu \mathrm{m}$ in the end-caps. On each side of the module, the sensors are bonded edge-to-edge so that the strips are daisy chained to form $\sim 12 \mathrm{~cm}$-long readout channels. Combining the measurements from both sides, a two-dimensional space-point is created. The spatial resolution achieved is $\sim 17 \mu \mathrm{m}$ in the $R \phi$ coordinate and $\sim 580 \mu \mathrm{m}$ in $Z$.

A binary readout is employed in the SCT modules. The front-end (FE) ASIC used for the readout of 128 channels from a single microstrip sensor is the Atlas Binary Chip ABCD3TA [2]. Fabricated in radhard DMILL technology, it comprises both analogue (pre-amplification, shaping and discrimination) and digital stages. The FE chips are mounted on hybrids, "centre-tapped" in the barrel modules and "end-tapped" in the end-caps. The hybrids are double-sided, with six ABCD3TA chips per side. Two dedicated optoASICS are used for the transmission of optical signals between the modules and the off-detector electronics. The DORIC4A chip receives trigger, clock and control commands through a PIN diode. The VDC chip drives VCSELs diodes for two optical links for the transmission of the data of each readout side of a SCT module to the off-module ReadOut Driver (ROD).

\section{Commissioning of the SCT}

The macro-assembly of the SCT was finalized in 2005. During 2006, combined tests were performed after the integration of the SCT together with the TRT in the ATLAS SR1 surface building at CERN [3] and before they were put in place in the ATLAS experimental cavern. The SCT+TRT barrel detectors were installed as one package in the ATLAS pit in August 2006, being signed-off in May 2007. End-caps A and C were inserted into the cavern in May and June 2007 respectively. The complete end-cap sign-off came in during January/February 2008.

Then the SCT took successfully part in the major data-taking milestones:

- Milestone week 6 (March 2008), with most of the ATLAS detectors plus all trigger levels.

- First proton beam circulating in the LHC (September $\left.10^{\text {th }} 2008\right)$. The SCT end-caps, operated at low bias voltage and high threshold, detected the muon showers produced in the forward direction from the interactions of the protons with the beam collimators.

- ATLAS global cosmic run (September/October 2008), a combined cosmic data-taking campaign including all ATLAS sub-systems (including the Pixel detector already signed-off) and different configurations (on/off) of the solenoid field. During this period $\sim 2 \times 10^{6}$ tracks were reconstructed in the SCT.

The commissioning tests focused in thorough testing the detector connectivity and services (electrical and optical connections, cooling system), together with an optimization and tuning of the front-end parameters through continous and refined calibration procedures.

The SCT is cooled by means of an evaporative system based on $\mathrm{C}_{3} \mathrm{~F}_{8}$. The fluid expands through capillaries remaining in saturation conditions. The residual liquid is evaporated by heaters located at the exhausts of the cooling lines. In order to reach $-10{ }^{\circ} \mathrm{C}$ in the SCT modules, $-25{ }^{\circ} \mathrm{C}$ is needed in the cooling loops. The major cooling problem happened in May 2008 with the failure of three out of six compressors of the ID cooling plant (common to Pixels and SCT) required to keep the low operating temperature. After the affected compressors were replaced, the cooling plant is up again since August 2008.

All but one cooling loops are running in the SCT. The failling loop located in Disk-9 (highest Z-position) of 
end-cap $\mathrm{C}$ is non-recoverable due to accessibility constraints. The consequence is that 13 end-cap modules will remain out-of service, corresponding to $0.3 \%$ of the total number of SCT modules.

Another problem encountered during the operation of the detector was the elevated infant mortality in the off-detector optical transmitter boards. Electro-Static Discharges (ESD) during the production phase appear to be the cause of the high failure rate. No problems have been found in the on-detector VCSELs opto-chips. The replacement operation of the damaged plug-ins was completed in summer 2009.

As of today, more than $99 \%$ of the SCT components (including cooling loops, modules, chips and strips) are fully operational.

The calibration procedure of the SCT modules is based on the injection of test charges with adjustable amplitude by means of the front-end internal calibration circuit. The discriminator compares the input charge with the threshold value and produces a binary hit if the signal is above the threshold. Analogue parameters like gain, threshold spread and noise are evaluated by scanning the discriminator threshold for a given input charge. The noise occupancy is defined as the probability for a strip to give a hit in a certain event only due to noise. Fig 3 shows the noise occupancy obtained for barrel and end-cap outer and middle modules. All calibrated chips are within the specification of a noise occupancy per strip less than $5 \times 10^{-4}$ at $1 \mathrm{fC}$ operating threshold.

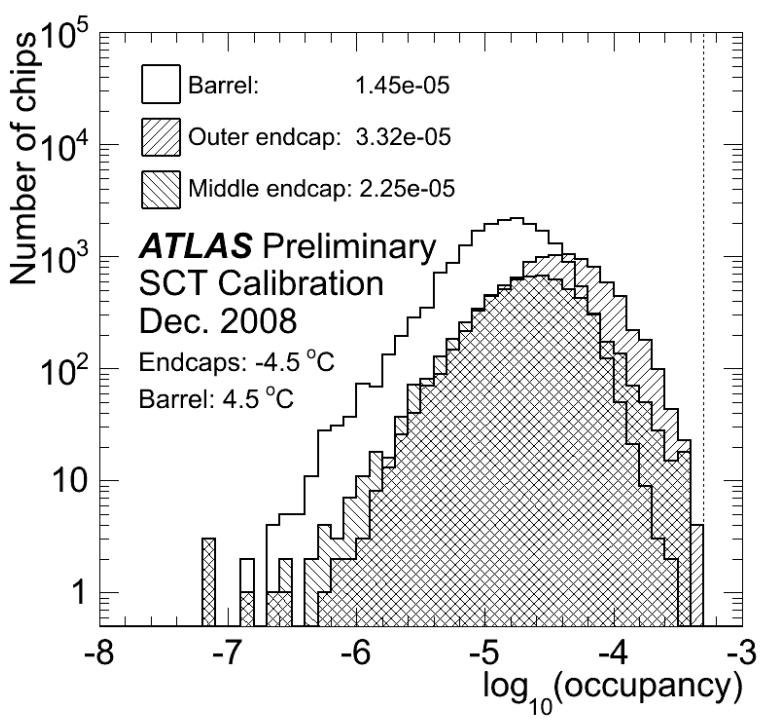

Figure 3: Noise occupancy in barrel and end-cap outer and middle SCT modules from calibration tests.

\section{Alignment}

The strategy for the alignment of the ID is based on track-based offline alignment algorithms complemented by a Frequency Scanning Interferometry (FSI) system in the barrel SCT. Different alignment algorithms [4, 5] have been successfuly implemented and tested in periods previous to the ATLAS combined cosmic runs. Most of the alignment algorithms are based on the minimization of a $\chi^{2}$ function defined from the residuals (difference between the prediction from a trackextrapolation and the real measurement in the detector) of each reconstructed track in a module. The alignment infrastructure is based on corrections performed at different levels, from entire sub-detectors (e.g. SCT barrel and end-caps) up to the single module level. Fig. 4 compares the unbiased residuals (hit of the module under consideration excluded from the track-fit) along the $R \phi$ coordinate before and after alignment. The improvement with respect to the nominal or as-built geometry (i.e., without any alignment correction applied) is significant. The distributions are smeared due to track extrapolation errors, several Gaussian-distributed sources of noise and alignment errors. The magnitude of the remaining misalignments can be extracted by comparison with Monte-Carlo events simulated with a perfectly aligned detector. The final alignment accuracy will be achieved with collision data.

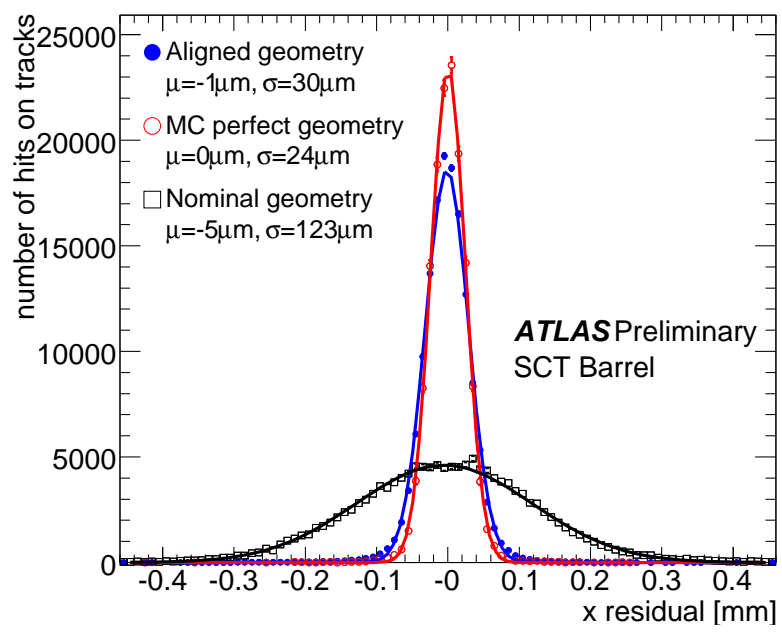

Figure 4: Residual distributions along the precision coordinate in the SCT barrel from real data for the nominal (without any alignment corrections) and aligned geometries. The corresponding residuals obtained from simulated cosmics with a perfect geometry are also included. The results (mean $\mu$ and sigma $\sigma$ ) of a Gaussian fit to each ditribution are shown. 


\section{Performance results}

\subsection{Tracking performance}

In ATLAS, the track parameters are given at the perigee, the point of closest approach of the trajectory to the $Z$-axis. The five track parameters are the signed transverse impact parameter $d_{0}$, polar angle $\phi_{0}$, longitudinal impact parameter $z_{0}$, azimuthal angle $\theta_{0}$ and charge over momentum magnitude $q / p$. Cosmic tracks crossing the entire ID have been split near the interaction point and fit separately. The two halves would correspond to collision-like tracks that can be compared to get an estimate of the resolution in the track parameters and as a test of the goodness of the alignment.

Fig. 5 shows the difference in the reconstructed $d_{0}$ between the two split tracks. After alignment, the distribution is well centered around zero and approaches that of the ideal detector.

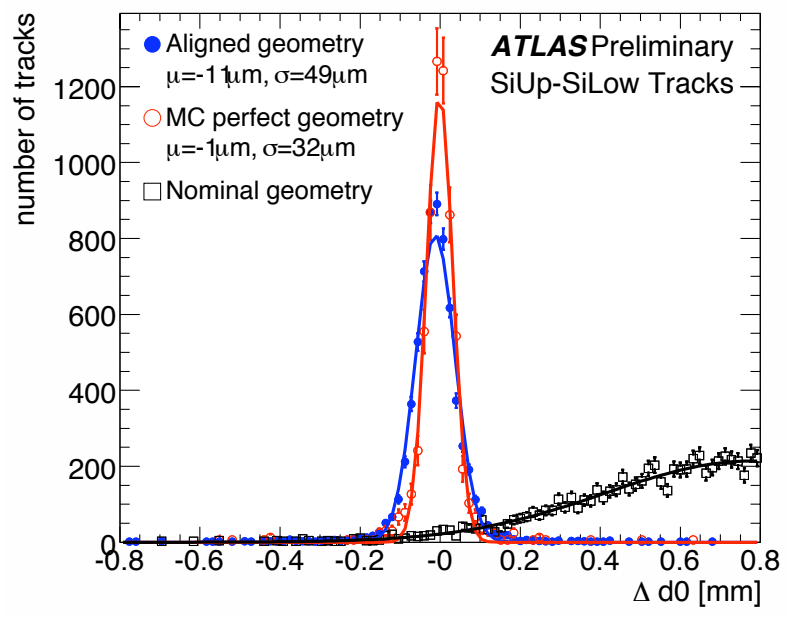

Figure 5: Difference in the reconstructed transverse impact parameter $d_{0}$ of two split cosmic tracks. Tracks are required to have $p_{T}>2 \mathrm{GeV} / \mathrm{c}$ and pass through the innermost pixel layer.

\subsection{Lorentz angle measurement}

In the presence of electric and magnetic fields, the carriers created in the silicon sensor after the passage of a charged particle drift along a direction at an angle $\theta_{L}$ (called the Lorentz angle) with respect to the electric field direction. The effect of the Lorentz angle is a shift of the collected charge. Depending on the incidence angle of the particles, the charges spread over several strips. Since this spread is minimal for an incidence angle equal to the Lorentz angle, $\theta_{L}$ can be measured by computing the minimum of the average strip cluster-size as a function of the incidence angle of the track to the module surface (see Fig. 6). The measured value of the Lorentz angle is found to be $\theta_{L}=3.93 \pm 0.03$ (stat) \pm 0.10 (syst) degrees for a bias voltage of $150 \mathrm{~V}$ and a sensor temperature of $\sim 278 \mathrm{~K}$, which agrees within systematic uncertainties with the model prediction of $\theta_{L}=3.69 \pm 0.26$ (syst) degrees at the same operating conditions. In the case of data recorded without solenoid field, the measured Lorentz angle is compatible with zero within systematic errors.

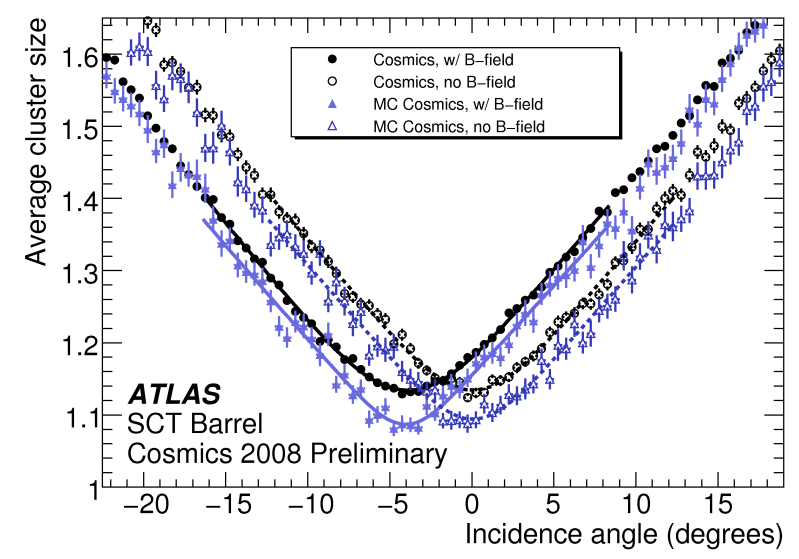

Figure 6: Lorentz angle measurement in the barrel SCT through determination of the minimum of the average cluster-size versus the track incidence angle.

\section{Conclusion}

The SCT has been installed in the ATLAS cavern and signed-off early in 2008. Several major problems (cooling, electro-optical) have been detected and solved. More than $99 \%$ of the SCT is fully operational. The detector is being run successfully in the major ATLAS cosmic data-taking combined periods since 2008 and is ready for the first LHC collisions expected to happen in November 2009.

\section{References}

[1] The ATLAS Collaboration, G.Aad et al. The ATLAS Experiment at the CERN Large Hadron Collider. JINST3 (2008) S08003.

[2] F. Campabadal et al. Design and performance of the ABCD3TA ASIC for the readout of silicon strip detectors in the ATLAS semiconductor tracker. NIMA 552(2005)292-328.

[3] E. Abat et al. Combined performance tests before installation of the ATLAS Semiconductor and Transition Radiation Tracking Detectors. JINST 3 (2008) P08003.

[4] The ATLAS Collaboration, A. Ahmad et al. Alignment of the Pixel and SCT modules for the 2004 ATLAS Combined Test Beam. JINST3 (2008) P09004.

[5] A. Bocci et al. TRT alignment for SRI cosmics and beyond. ATL-INDET-PUB-2007-009 (2007). 